\title{
Model-Informed Approaches for Alternative Aripiprazole Dosing Regimens and Missed Dose Management: Towards Better Adherence to Antipsychotic Pharmacotherapy
}

\author{
Jun-Yi Wu ${ }^{1} \cdot G u o Y^{2} \cdot G u o-F u ~ L i{ }^{1,2}(0)$ \\ Published online: 26 June 2018 \\ (c) Springer International Publishing AG, part of Springer Nature 2018
}

Systems pharmacology and pharmacometric modeling and simulation have become increasingly important in accelerating drug development and regulatory approval [1-4]; nevertheless, their use in precision pharmacotherapy and management of missed doses remain scarce [5]. Failure in the management of missed doses under various scenarios during treatment with antipsychotic medications might lead to a relapse of schizophrenia. In this issue, Hard and colleagues present a pharmacometric study of aripiprazole to characterize aripiprazole disposition following intramuscular (IM) administration of a nano-crystalline milled dispersion of aripiprazole lauroxil $\left(\mathrm{AL}_{\mathrm{NCD}}\right)$ and $\mathrm{AL}$ and oral administration of aripiprazole, and to explore the feasibility of a 1-day initiation regimen using $\mathrm{AL}_{\mathrm{NCD}}$ plus oral aripiprazole alternative to the 21-day initiation regimen of $\mathrm{AL}$ alone, and to inform strategies to manage missed doses of $\mathrm{AL}$ under different treatment scenarios [6]. The research might serve as a basis for alternative aripiprazole dosing regimens and missed dose management, aiming at better care for patients with schizophrenia and adherence to aripiprazole treatment.

The first requirement of model-based dosing optimization is a well-established therapeutic window or target exposures associated with approved dosing regimen tested in pivotal clinical studies, aiming to maximize the therapeutic benefit and minimize exposure-related toxicities. Although dissimilar therapeutic windows of aripiprazole were documented in

This comment refers to the article available at https://doi. org/10.1007/s13318-018-0488-4.

Guo-Fu Li

guofu.g.li@gmail.com

1 Center for Drug Clinical Research, Shanghai University of Chinese Medicine, No. 1200 Cailun Rd, Shanghai 201203, China

2 College of Clinical Medicine, Yangzhou University, Jiangsu, China different reports or clinical guidelines [7-9], Hard et al. [6] rationally adopted the plasma concentration range associated with therapeutic doses of AL using the 21-day initiation regimen as the reference aripiprazole plasma levels that should be matched following alternative initiation regimen of aripiprazole.

Before a pharmacometric model can be applied to assess the potential changes in exposure or response under complex clinical scenarios, the model should be adequately qualified, which should follow the "learn and confirm" paradigm. Ideally, the model should go through internal qualification to determine the adequacy of the model for current data and external qualification to evaluate performance using other data from a different population sample. Furthermore, the model may be refined in the "learn and confirm" cycles when new clinical data become available. In the study by Hard et al. [6], the model was built on the basis of data from three individual studies [Study 1 (ALK9072-B101), Study 3 (ALK9072-B103), and Study 2 (ALK9072-B102)] in a sequential manner, and then updated based on the pooled data from the three studies plus another Phase 1 study conducted earlier. The model performance was judged mainly by posterior prediction-corrected visual predictive checks and goodness-of-fit assessments. Surprisedly, the final population pharmacokinetic model overpredicted aripiprazole exposure following multiple oral dose administration of aripiprazole in Study 2. The final model was, therefore, reupdated to characterize the high variability in aripiprazole concentrations by accounting for a subgroup of patients with lower exposure among those receiving the 21-day initiation regimen in Study 2 via a mixture model. After then, the updated final model was used for simulations to evaluate the feasibility of 1-day initiation regimen involving $\mathrm{AL}_{\mathrm{NCD}}$ and inform the strategies of manage missed doses using 1-day single injection of $\mathrm{AL}_{\mathrm{NCD}}$. In spite of lack of external qualification, the model development processes of aripiprazole generally followed the "learn and confirm" paradigm. 


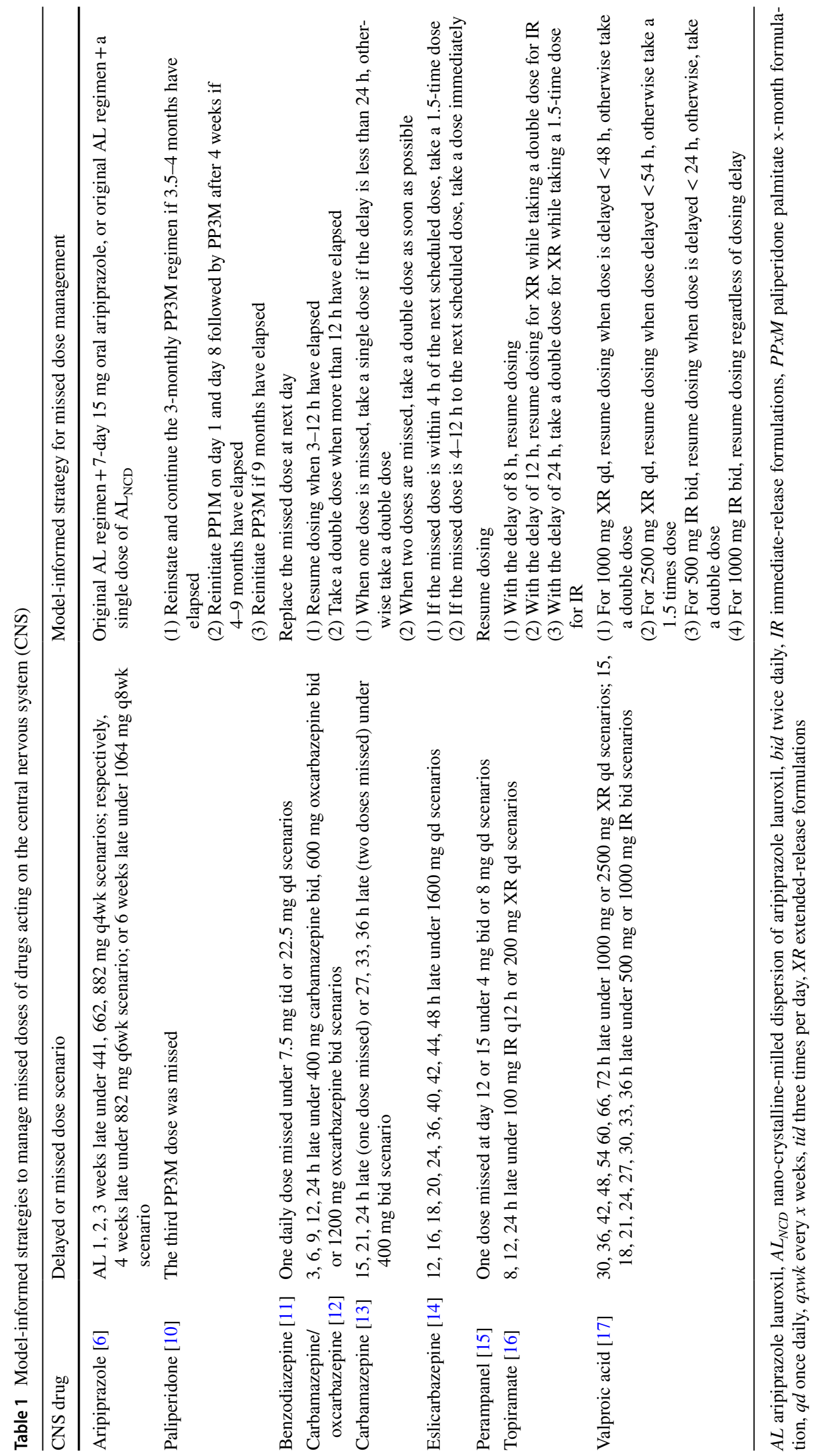


Missed or delayed doses of antipsychotic medications are commonly encountered in clinical practice, leading to suboptimal therapeutic outcomes. For patients with schizophrenia or another central nervous system (CNS) disorders, subtherapeutic plasma levels under missed dose scenarios could result in a relapse or symptoms out of control. To prevent or minimize the risk of relapse, missed doses should be appropriately managed. Unfortunately, the impact of various missed dose scenarios on drug exposure and efficacy is not likely to be sufficiently investigated in pivotal clinical trials. Instead, system pharmacology and pharmacometric modeling and simulation offer great opportunities to better understand the in vivo behavior of medications in clinically challenging situations. Table 1 compiled some selected examples of model-informed strategies to manage missed doses of antipsychotic medicines or other CNS drugs, such as paliperidone, benzodiazepine, carbamazepine.

One of the limitations pointed out by Hard and colleagues [6] is that simulated data solely inform the alternative 1-day initiation regimen and the missed dose management. External clinical data should further reassure the clinical impact of the alternative initiation regimen and strategies to manage missed doses. In this regard, we believe, well-qualified pharmacometric models could serve as the basis for dosing optimization in the clinical setting. We are optimistic about clinical applications of their model since population pharmacokinetic models can be verified by routinely collected data through therapeutic drug monitoring.

\section{Compliance with Ethical Standards}

Funding No funding was received for this work.

Conflict of interest The authors have no conflicts of interest to declare.

\section{References}

1. Derendorf H, Lesko LJ, Chaikin P, Colburn WA, Lee P, Miller R, et al. Pharmacokinetic/pharmacodynamic modeling in drug research and development. J Clin Pharmacol. 2000;40(12):1399-418.

2. Meibohm B, Derendorf H. Pharmacokinetic/pharmacodynamic studies in drug product development. J Pharm Sci. 2002;91(1):18-31.

3. Yu G, Wang DX, Li GF, Zhou HH. The role of a meal on gastrointestinal toxicity and maximum tolerated dose. Lancet Oncol. 2016;17(1):e2-3.
4. Li GF, Zheng QS. Modeling drug disposition and drug-drug interactions through hypothesis-driven physiologically based pharmacokinetics: a reversal translation perspective. Eur J Drug Metab Pharmacokinet. 2018;43(3):369-71.

5. Darwich AS, Ogungbenro K, Vinks AA, Powell JR, Reny JL, Marsousi N. Why has model-informed precision dosing not yet become common clinical reality? Lessons from the past and a roadmap for the future. Clin Pharmacol Ther. 2017;101(5):646-56.

6. Hard ML, Wehr AY, Sadler BM, Mills RJ, von Moltke L. Population pharmacokinetic analysis and model-based simulations of aripiprazole for a 1-day initiation regimen for the long-acting antipsychotic aripiprazole lauroxil. Eur J Drug Metab Pharmacokinet. 2018. https://doi.org/10.1007/s13318-018-0488-4.

7. Raoufinia A, Baker RA, Eramo A, Nylander AG, Landsberg W, Kostic D, et al. Initiation of aripiprazole once-monthly in patients with schizophrenia. Curr Med Res Opin. 2015;31:583-92.

8. Raoufinia A, Peters-Strickland T, Nylander AG, Baker RA, Eramo A, Jin N, et al. Aripiprazole once-monthly $400 \mathrm{mg}$ : comparison of pharmacokinetics, tolerability, and safety of deltoid versus gluteal administration. Int J Neuropsychopharmacol. 2017;20:295-304.

9. Jann MW, Penzak SR. Long-acting injectable second-generation antipsychotics: an update and comparison between agents. CNS Drugs. 2018;32(3):241-57.

10. Magnusson M, Samtani M, Plan E, Jonsson E, Rossenu S, Russu A, et al. Dosing and switching strategies for paliperidone palmitate 3-month formulation in patients with schizophrenia based on population pharmacokinetic modeling and simulation, and clinical trial data. CNS Drugs. 2017;31(4):273-88.

11. Riss J, Cloyd J, Gates J, Collins S. Benzodiazepines in epilepsy: pharmacology and pharmacokinetics. Acta Neurol Scand. 2008;118(2):69-86.

12. Ahmad A, Garnett WR. Carbamazepine extended-release capsules vs. oxcarbazepine: computer simulations of the effect of missed doses on drug plasma concentrations. Curr Med Res Opin. 2005;21(9):1363-8.

13. Garnett WR, McLean AM, Zhang Y, Clausen S, Tulloch SJ. Simulation of the effect of patient nonadherence on plasma concentrations of carbamazepine from twice-daily extended-release capsules. Curr Med Res Opin. 2003;19(6):519-25.

14. Sunkaraneni S, Blum D, Ludwig E, Chudasama V, Fiedler-Kelly J, Marvanova M, et al. population pharmacokinetic evaluation and missed-dose simulations for eslicarbazepine acetate monotherapy in patients with partial-onset seizures. Clin Pharmacol Drug Dev. 2018;7(3):287-97.

15. Gidal BE, Majid O, Ferry J, Hussein Z, Yang H, Zhu J, et al. The practical impact of altered dosing on perampanel plasma concentrations: pharmacokinetic modeling from clinical studies. Epilepsy Behav E\&B. 2014;35:6-12.

16. Brittain S, Wheless J. Pharmacokinetic simulations of topiramate plasma concentrations following dosing irregularities with extended-release vs. immediate-release formulations. Epilepsy Behav E\&B. 2015;52((Pt A)):31-6.

17. Ahmad A. A nomogram for valproic acid and the effect of missed doses. Dissertation, Virginia Commonwealth Univ, Richmond, VA; 2005. 